\title{
Enhanced Cuckoo Search Optimization and Hybrid Firefly Artificial Neural Network Algorithm for Cyberbullying Detection on Twitter Dataset
}

\author{
Sherly T.T ${ }^{1}$, Dr. B. Rosiline Jeetha ${ }^{2}$ \\ ${ }^{1}$ Research Scholar, PG and Research Department of Computer Science, Dr. N.G.P. Arts and Science College, \\ Coimbatore, India \\ ${ }^{2}$ Research Guide, PG and Research Department of Computer Science, Dr. N.G.P. Arts and Science College, \\ Coimbatore, India
}

\section{Article Info \\ Volume 7, Issue 4 \\ Page Number: 455-470}

\section{Publication Issue :}

July-August-2021

\section{Article History}

Accepted : 01 Aug 2021

Published : 08 Aug 2021

\section{ABSTRACT}

With the exponential increase of social media users, cyberbullying has been emerged as a form of bullying through electronic messages. Cyberbullying detection is generally in social networks like Twitter is one of the focussed research area. Cyberbullying is serious and widespread issues affecting increasingly more Internet users. Text mining tools are detecting cyber bullying and deal with several issues. However the existing system has issue with time consumption and inaccurate Cyberbullying detection results for the given Twitter dataset. To avoid the above mentioned issues, in this work, Enhanced Cuckoo Search optimization (ECSO) and Hybrid Firefly Artificial Neural Network (HFANN) algorithm is proposed. The proposed system contains three main phases are such as preprocessing, feature subset selection and classification. The preprocessing is done by using $\mathrm{k}$-means algorithm for reducing the noise data from the given Twitter dataset. It handles the missing features and redundancy features through $\mathrm{k}$-means centroid values and $\min \max$ normalization respectively. It is used to increase the classification accuracy more effectively. The pre-processed features are taken into feature selection process for obtaining more informative features from the Twitter dataset. It is performed by using ECSO algorithm and the objective function is used to compute the relevant and important feature based on the best fitness values. Then the HFANN algorithm is applied for classification through training and testing model. It classifies the features more accurately using best fireflies rather than the previous algorithms. The experimental result proves that the proposed ECSO+HFANN algorithm provides better classification performance in terms of lower time complexity, higher precision, recall, f-measure and accuracy than the existing algorithms.

Keywords : Cyberbullying detection, Twitter dataset, k-means algorithm, Enhanced Cuckoo Search optimization (ECSO), Hybrid Firefly Artificial Neural Network (HFANN) 


\section{INTRODUCTION}

Text mining has turned into a vital research zone and it discloses new obscure data, by consequently removing data from various composed assets. A key element is the linking together of the extracted information together to form new facts or new hypotheses to be explored further by more conventional means of experimentation. Text mining is different from what are familiar with in web search [1]. In search, the user is typically looking for something that is already known and has been written by someone else. The problem is pushing aside all the material that currently is not relevant to your needs in order to find the relevant information. In text mining, the goal is to discover unknown information, something that no one yet knows and so could not have yet written down.

Cyberbullying can be defined as aggressive, intentional actions performed by an individual or a group of people via digital communication methods such as sending messages and posting comments against a victim [2] [3]. Different from traditional bullying that usually occurs at school during face to face communication, Cyberbullying on social media can take place anywhere at any time. For bullies, they are free to hurt their peers' feelings because they do not need to face someone and can hide behind the Internet. For victims, they are easily exposed to harassment since all of us, especially youth, are constantly connected to Internet or social media.

One of the most visited social networking sites by millions of users is twitter where they share their opinion about various domains like politics, brands, products, celebrities etc. Twitter is an immensely popular micro-blogging network where people post short messages of 140 characters called tweets [4]. It has over 100 million active users who post about 200 million tweets every day. Some of the Twitter specific features we use are tweet content and its characteristics like length, hashtags, and mentions. Other Twitter features used are the characteristics of the Twitter user posting the tweet such as age of the account, number of tweets, and the follower-followee ratio. These twitter specific features coupled with URL based features prove to be a strong mechanism to detect phishing tweets.

Twitter endeavours to provide a space for people to express themselves freely. Additionally, provides a medium for users to report any sort of abusive content on the platform. The user can include multiple tweets in the same report, helping Twitter to improve its context, while inspecting the problems to get them resolved sooner. In addition, a user can block, mute or unfollow other unwanted users. In [5], Özel et al. gathered data from Twitter and Instagram written in Turkish and then applied decision tree (C4.5), SVM, Multinomial Naïve Bayes, and K Nearest Neighbors (KNN) to detect Cyberbullying. As per observation, accuracy improves when they considered both words and emoticons in the text messages as features. Naïve Bayes outperformed all other algorithms in their experiment, and it achieved 84\% accuracy. Fig 1 shows the example of Twitter data.

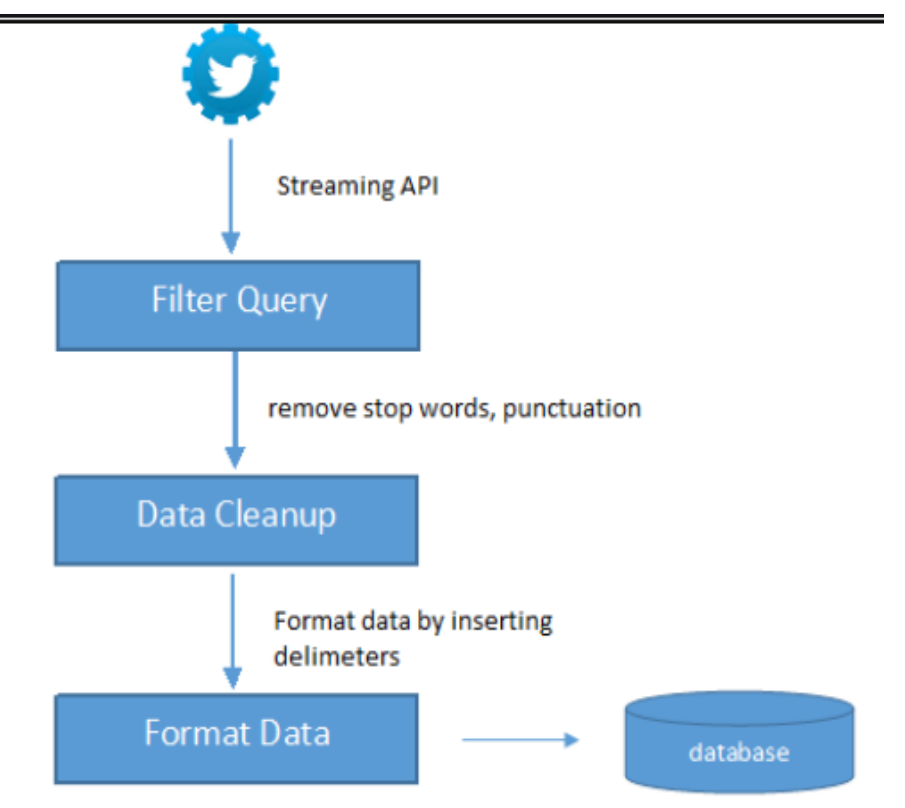


Sherly T. T. et al Int. J. Sci. Res. Comput. Sci. Eng. Inf. Technol, July-August-2021, 7 (4) : 455-470

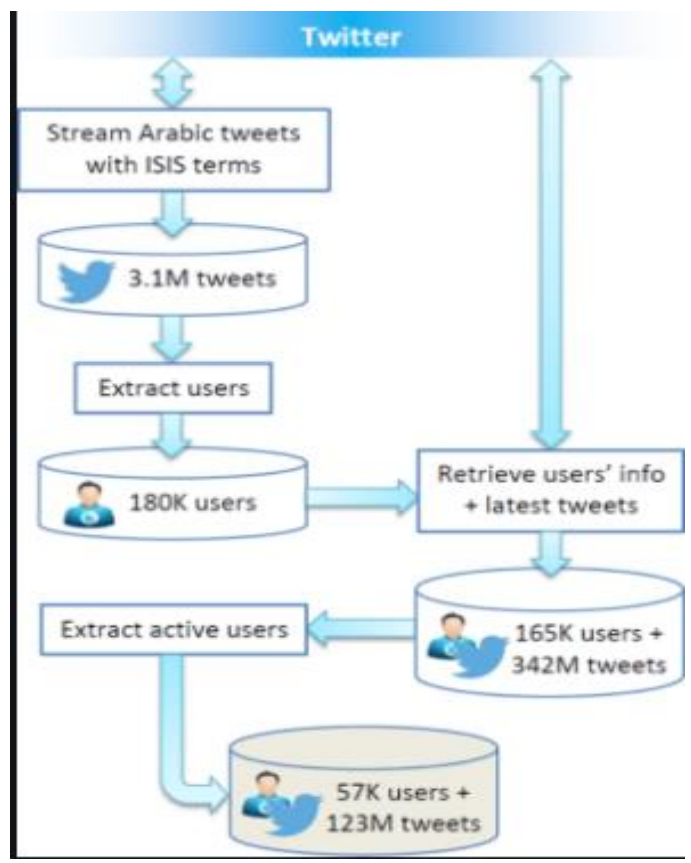

Fig 1. example of Twitter data

The features selection process is an important stage finding efficient features (more discriminant) and improving the quality of datasets (better and faster results). Different features are extracted from the given datasets have been evaluated to achieve better representation. The accuracy of the Cyberbullying classifier not only depends on the classification algorithm but also on the feature selection method. Selection of irrelevant and inappropriate features may confuse the classifier and lead to incorrect results for the given Twitter dataset. The optimization based solution to this problem is feature selection which is by removing the irrelevant and redundant features from the original dataset [6]. Classification is the process of predicts categorical labels. It is used to classify the data based on the training set and the values in a classifying attribute. SVM is supervised pattern classification which has been applied to Twitter dataset to classify. SVM also finds a nonlinear decision function in the input space by mapping the data into a higher dimensional feature space and separating by means of a maximum margin hyper plane. The system automatically identifies a subset of informative points called support vectors and uses them to represent the separating hyper plane which is sparsely a linear combination of these points [7].

The main problem of this research work is the Cyberbullying detection on Twitter dataset. There are several research and methodologies introduced but the Twitter dataset accuracy is not achieved significantly. The existing approaches has drawback with time consumption and inaccurate Cyberbullying detection results. To overcome the abovementioned issues, in this research, ECSO+ HFANN is proposed to improve the overall system performance. The main contribution of this research is preprocessing, feature selection using ECSO algorithm and Cyberbullying detection using HFANN. The proposed method is used to provide more accurate results using effective algorithms for the given Twitter dataset.

\section{RELATED WORK}

In [8], Al-Garadi et al (2016) introduced set of unique features derived from Twitter; network, activity, user, and tweet content, based on these feature, we developed a supervised machine learning solution for detecting Cyberbullying in the Twitter. An evaluation demonstrates that our developed detection model based on our proposed features, achieved results with an area under the receiver-operating characteristic curve of 0.943 and an f-measure of 0.936 . These results indicate that the proposed model based on these features provides a feasible solution to detecting Cyberbullying in online communication environments. Finally, we compare result obtained using our proposed features with the result obtained from two baseline features. The comparison outcomes show the significance of the proposed features.

In [9], León-Paredes et al (2019) deploys a Spanish cyberbullying prevention system (SPC), which relies on Natural Language Processing (NLP) methods and different machine learning techniques (Naive Bayes, Support Vector Machine and Logistic Regression), using Twitter as the basis for the extraction of 
knowledge bases or corpus. Several precision metrics and variable corpus sizes are used for the training. The learning results reach a maximum accuracy of $93 \%$, verified through the application of three study cases

In [10], Zhang et al (2019) focus on the Japanese text on Twitter and construct an optimal model for automatic detection of Cyberbullying by extracting multiple textual features and investigating their effects with multiple machine learning models. The experimental evaluation shows that the best model with predictive textual features is able to obtain an accuracy of over $90 \%$.

In [11], Balakrishnan et al (2020) deals with automatic Cyberbullying detection mechanism tapping into Twitter users' psychological features including personalities, sentiment and emotion. User personalities were determined using Big Five and Dark Triad models, whereas machine learning classifiers namely, Naïve Bayes, Random Forest and J48 were used to classify the tweets into one of four categories: bully, aggressor, spammer and normal. The Twitter dataset contained 5453 tweets gathered using the hashtag \#Gamergate, and manually annotated by human experts. Selected Twitter-based features namely text, user and network-based features were used as the baseline algorithm. Results show that Cyberbullying detection improved when personalities and sentiments were used, however, a similar effect was not observed for emotion. A further analysis on the personalities revealed extraversion, agreeableness, neuroticism and psychopathy to have greater impacts in detecting online bullying compared to other traits. Key features were identified using the dimension reduction technique, and integrated into a single model, which produced the best detection accuracy. The paper describes suggestions and recommendations as to how the findings can be applied to mitigate Cyberbullying

In [12], Dash et al (2010) introduced K-means is a well known partitioning based clustering technique that attempts to find a user specified number of clusters represented by their centroids. But its output is quite sensitive to initial positions of cluster centers. Again, the number of distance calculations increases exponentially with the increase of the dimensionality of the data. Hence, in this paper we proposed to use the Principal Component Analysis (PCA) method as a first phase for K-means clustering which will simplify the analysis and visualization of multi dimensional data set. Here also, we have proposed a new method to find the initial centroids to make the algorithm more effective and efficient. By comparing the result of original and new approach, it was found that the results obtained are more accurate, easy to understand and above all the time taken to process the data was substantially reduced

In [13], Salesi et al (2017) focused to improve the weaknesses of the Cuckoo Search algorithm, this paper proposes a pseudobinary mutation neighbourhood search procedure embedded in a new binary version of the Cuckoo Search algorithm. The proposed Extended Binary Cuckoo Search algorithm has been designed for the task of feature selection, and thus aims to minimise the number of selected features (such that only the best features are retained in the subset) whilst maximising the classification accuracy. Based on these criteria, a new objective function is proposed which considers the number of features in the subset as well as the classification accuracy when searching for the best subset of features. To measure the classification accuracy when using a set of candidate features, the Support Vector Machine classifier is utilised. Experiments were conducted with the proposed Extended Binary Cuckoo Search optimisation applied to biomedical datasets and the results demonstrated the superiority of the proposed algorithm against three other natureinspired algorithms, namely the Binary Ant Colony Optimisation, Binary Genetic Algorithm, and Binary Particle Swarm Optimisation. Moreover, the experiments revealed that when using the proposed function, higher classification accuracy is achieved using a fewer number of features, as opposed to using 
the standard classification accuracy function which needed more features to achieve comparable accuracy. In [14], Abd El Aziz et al (2018) suggested modified cuckoo search algorithm with rough sets is presented to deal with high dimensionality data through feature selection. The modified cuckoo search algorithm imitates the obligate brood parasitic behavior of some cuckoo species in combination with the Lévy flight behavior of some birds. The modified cuckoo search uses the rough sets theory to build the fitness function that takes the number of features in reduct set and the classification quality into account. The proposed algorithm is tested and validated benchmark on several benchmark datasets drawn from the UCI repository and using different evaluation criteria as well as a further analysis is carried out by means of the Analysis of Variance test. In addition, the proposed algorithm is experimentally compared with the existing algorithms on discrete datasets. Finally, two learning algorithms, namely K-nearest neighbors and support vector machines are used to evaluate the performance of the proposed approach. The results show that the proposed algorithm can significantly improve the classification performance.

In [15], Emary et al (2015) used the system for feature selection based on firefly algorithm (FFA) optimization is proposed. Data sets ordinarily include a huge number of attributes, with irrelevant and redundant attributes. Redundant and irrelevant attributes might reduce the classification accuracy because of the large search space. The main goal of attribute reduction is to choose a subset of relevant attributes from a huge number of available attributes to obtain comparable or even better classification accuracy from using all attributes. A system for feature selection is proposed in this paper using a modified version of the firefly algorithm (FFA) optimization. The modified FFA algorithm adaptively balances the exploration and exploitation to quickly find the optimal solution. FFA is a new evolutionary computation technique, inspired by the flash lighting process of fireflies. The FFA can quickly search the feature space for optimal or near-optimal feature subset minimizing a given fitness function. The proposed fitness function used incorporates both classification accuracy and feature reduction size. The proposed system was tested on eighteen data sets and proves advance over other search methods as particle swarm optimization (PSO) and genetic algorithm (GA) optimizers commonly used in this context using different evaluation indicators.

In [16], Ghiassi et al (2013) introduced an approach to supervised feature reduction using n-grams and statistical analysis to develop a Twitter-specific lexicon for sentiment analysis. We augment this reduced Twitter-specific lexicon with brand-specific terms for brand-related tweets. We show that the reduced lexicon set, while significantly smaller (only 187 features), reduces modelling complexity, maintains a high degree of coverage over our Twitter corpus, and yields improved sentiment classification accuracy. To demonstrate the effectiveness of the devised Twitter-specific lexicon compared to a traditional sentiment lexicon, we develop comparable sentiment classification models using SVM. We show that the Twitter-specific lexicon is significantly more effective in terms of classification recall and accuracy metrics. We then develop sentiment classification models using the Twitter-specific lexicon and the DAN2 machine learning approach, which has demonstrated success in other text classification problems. We show that DAN2 produces more accurate sentiment classification results than SVM while using the same Twitter-specific lexicon.

\section{PROPOSED METHODOLOGY}

In this research work, the ECSO+ HFANN algorithm is proposed to improve the Cyberbullying detection results on the Twitter dataset. The proposed system contains preprocessing using $\mathrm{k}$-means clustering algorithm, feature selection using ECSO algorithm and Cyberbullying detection process using HFANN algorithm. 


\subsection{Preprocessing using k-means algorithm}

In this work, preprocessing is done by using $\mathrm{k}$-means clustering algorithm to increase the Cyberbullying detection accuracy. Twitter data is noisy and it contains emoticons, folksonomies, slangs and censored words [24]. Also it consists of short message texts. It is important to extract as much meaningful information as possible. K-means is an effective clustering technique used to separate similar data into groups based on initial centroids of clusters [17]. It uses the concept of Euclidean distance to calculate the centroids of the clusters. Starting from a random partitioning, the algorithm repeatedly (i) computes the current cluster centers (i.e. the average vector of each cluster in data space) and (ii) reassigns each data item to the cluster whose centre is closest to it. It terminates when no more reassignments take place. By this means, the intra-cluster variance, that is, the sum of squares of the differences between data features and their associated cluster centers is locally minimized. k-means' strength is its runtime, which is linear in the number of data elements and its ease of implementation. In this work, the cluster number is kept equal to the number of classes.

\section{Algorithm 1: k-means algorithm}

1. Choose a number of clusters $\mathrm{k}$ from Twitter Dataset (TD)

Initialize cluster centers $\mu 1, \ldots \mu \mathrm{k}$

2. Pick k data (tweets) points and set cluster centers to these points

3. Randomly assign points to clusters and take means of clusters

4. For each data point, compute the cluster center it is closest to and calculate the distance measure for finding the missing values using given below formula

$d(i, j)=\sqrt{\sum_{i=1}^{n}\left(x_{i}-y_{i}\right)^{2}}$

5. Where $x_{i}$ and $y_{i}$ are two points in Euclidean nspace
6. Find redundancy features using min-max

normalization $\mathrm{v}^{\prime}=\frac{\mathrm{v}-\min (\mathrm{D})}{\max (\mathrm{D})-\min (\mathrm{D})}$

Where min and max is minimum and maximum values for the features in the $\mathrm{D}$

7. Assign the data point to this cluster

8. Re-compute cluster centers (mean of data points in cluster)

9. Stop when there are no new re-assignments.

From the original Twitter dataset the instances having missing attributes are separated from the dataset. The dataset is divided into two sets where one set contains complete instances that do not contain any missing values and the other set contains incomplete instances which contains missing values. $\mathrm{K}$-means clustering is applied on complete instances set to obtain clusters of complete instances. Hence, the instances are taken one by one and the missing attributes are filled with their possible values. K-means clustering is applied to the dataset from the resultant clusters, the newly added instance is validated that whether it is been clustered in the correct class or not. If it is in the correct cluster, then the assigned value is made as permanent then the procedure is continued with the next instance. If it is in the wrong cluster then the next possible value will be assigned and compared till it founds the value which put the instance in the correct cluster. The redundancy values are determined and reduced by using min-max normalization [18]. In pre-processing, K-means method performs uppercase to lowercase conversion, replacement of URLs, removal of extra symbols including punctuation marks and hashtags, conversion of emoticons into the best suitable words, stop words removal, stemming of words into their root form. Thus the preprocessing method is used to improve the Cyberbullying classification accuracy effectively by using k-means algorithm. The overall block diagram is shown in Fig 2 
Sherly T. T. et al Int. J. Sci. Res. Comput. Sci. Eng. Inf. Technol, July-August-2021, 7 (4) : 455-470

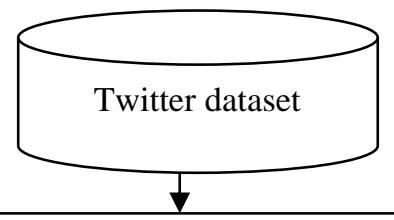

Data preprocessing using k-means algorithm

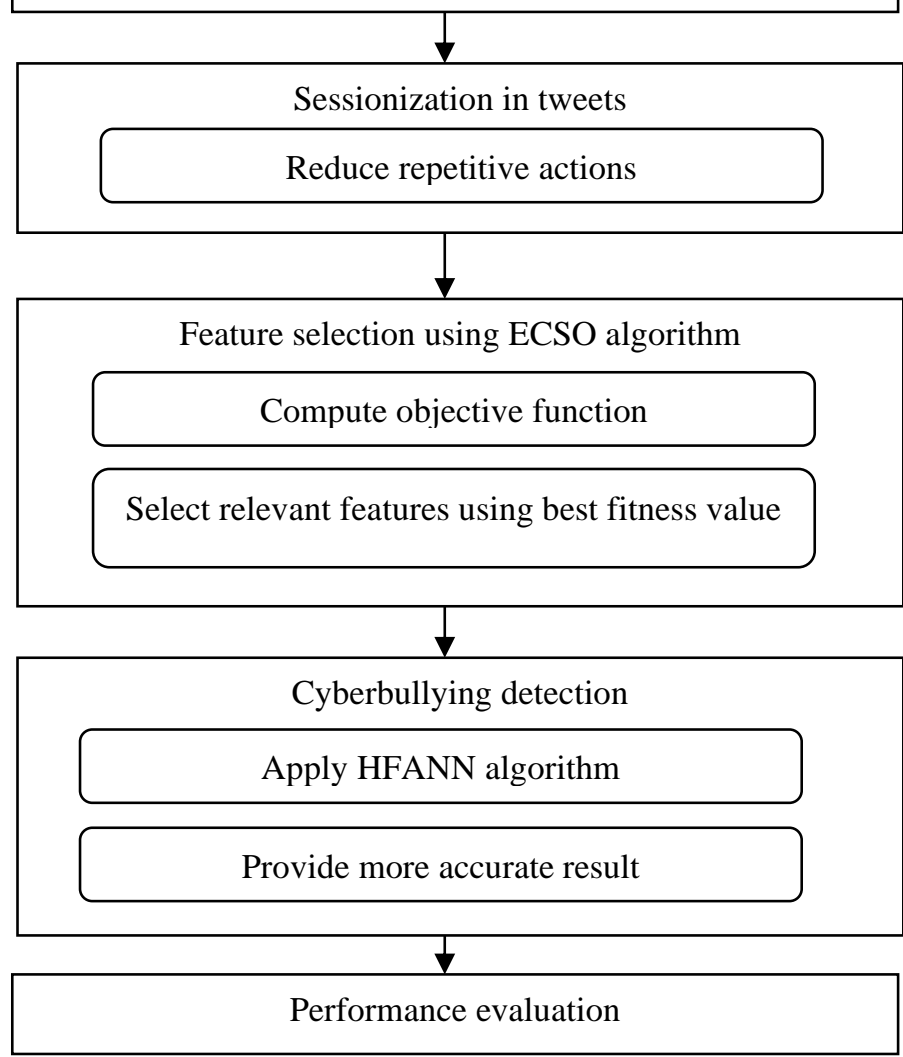

Fig 2. Overall block diagram of the proposed system

\subsection{Sessionization}

Cyberbullying usually involves repetitive actions, thus, it aims to study users' tweets over time. In [19], who consider a lower threshold of comments for media sessions extracted from Instagram to be presented on the annotation process and it creates, for each user, sets of time-sorted tweets (sessions) by grouping tweets posted close to each other in time. First, it removes users who are not significantly active, i.e., tweeting less than five times in the 3-month period. Then, it uses a session-based model where, for each session $\mathrm{Si}$, the inter-arrival time between tweets does not exceed a predefined time threshold tl. The minimum, median, and maximum length of the resulting sessions (in terms of the number of their included tweets) for the hate-related dataset are, respectively, 12, 22, and 2.6k tweets. For the baseline set of tweets, they are 5,44 , and $1.6 \mathrm{k}$ tweets.

\subsection{Feature selection using Enhanced Cuckoo Search optimization (ECSO)}

Feature selection is done by using Enhanced Cuckoo Search optimization (ECSO) algorithm which is used to choose the relevant features from the tweets. By using ECSO algorithm, optimal feature selection is done through best fitness values. In this section, the feature selection is performed by using ECSO algorithm for selecting important and more informative features from the given Twitter dataset. Cuckoo search is one of the recent nature-inspired metaheuristic algorithms which are used for producing optimal solutions. Cuckoos are attractive birds, because of the beautiful sounds they can make and their aggressive reproductive strategy. A few species such as the Ani and Guira cuckoos lay their eggs in communal nests and they may remove others' eggs to increase the hatching probability of their own eggs. CSO is based on the brood parasitism of few cuckoo species, by laying their eggs in the nest of other host birds. If a host bird identifies the eggs, which are not their own, it will throw these unknown eggs away or simply abandon its nest and built a new nest elsewhere [20]. Each egg in the nest represents a solution, and a cuckoo eggs represents a new solution. If the new solution (cuckoo) is better than old then replaces the solution which is so good in the nest. This CSO algorithm is enhanced by the Levy flights rather than by isotropic random walks. Fig 3 shows the Cuckoo's Habitat 


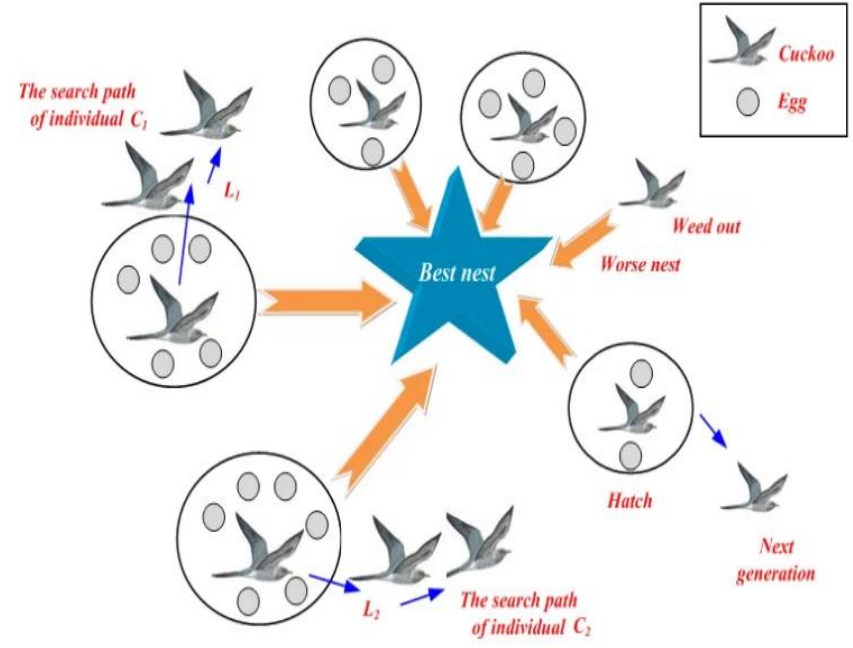

Fig 3. Cuckoo's Habitat

The following three idealized rules for describing standard cuckoo search have been used

- At a time each cuckoo lays one egg and dumps at a randomly chosen nest.

- The best nests with high quality eggs will be passed over to the next generations.

- Number of available host nests are fixed and the cuckoo's egg is discovered by the host bird with a probability belongs to $(0,1)$. The host bird can do either get rid of the egg or just abandon the nest and build a completely new nest.

This last assumption can be approximated by a fraction pa of the $\mathrm{n}$ host nests that are replaced by new nests (with new random solutions). CSO is very simple and has extensive search space. It uses the levy flight to global search instead of standard random walk, which makes CSO to explore the search space more efficiently.

CSO is improved through different types of host nest with multiple eggs [21]. In general cuckoo selects three types of nests for laying their eggs. The common cuckoo selects a group of host nests with egg characteristics similar to their own. Others cuckoo selects a group of host nest with egg characteristics dissimilar to their own. Some other species of cuckoo lay cryptic egg, which are dark in colour when the eggs of their host birds are light. This trick is used to hide the eggs from the host and evolve in cuckoos that parasite host with dark, domed nests.

\section{Initial Population}

In this work, each egg represents a possible set of features that are selected and used to classify the tweets correctly. The tweets (features) are selected from the top-m ranked features obtained from the statistical measures over the Twitter dataset.

\section{Finding new solutions and Levy flight}

ECSO based feature selection method makes use of levy flight for finding the new solutions from Equation (4). Some of the new solutions should be generated by a levy walk around the best solution obtained so far, this will speed up the local search. By using Levy flight, the new solution is $x_{i}^{(t+1)}$ is produced for cuckoo $i$, and it is given below

$\mathrm{x}_{\mathrm{i}}^{(\mathrm{t}+1)}=\mathrm{x}_{\mathrm{i}}^{(\mathrm{t})}+\mathrm{C} \oplus \operatorname{Levy}(\mathrm{s}, \lambda)$

$t$ is the step size. The step length follows the Levy distribution

$\operatorname{Levy}(\mathrm{S}, \lambda) \sim \mathrm{s}^{-\lambda}, \quad 1<\lambda \leq 3$

\section{Crossover and Mutation}

- Cuckoo type is common cuckoo then crossover is used to create two eggs in the nest and choose the best one among them.

- Cuckoo type is European cuckoo then creates two eggs using crossover with uniform mutation operator and choose the best one among them.

- Otherwise eggs (cryptic) are created with random solution.

\section{Fitness function}

The fitness function plays a vital role in the selection process. By using the best fitness function values, the prominent tweets (features) are selected from the Twitter dataset effectively. Classification accuracy plays a major role in the process of Twitter classification using significant features from the feature vector. Here, the classification accuracy of Minimum distance classifier is used as the fitness function of ECSO. Hence, both relevance and 
redundancy are included in the fitness function to guide ECSO to search for the best feature, which can be represented by Equation

$$
\text { fitness }(f)=\operatorname{accuracy}(f)
$$

Accuracy $(\mathrm{f})$ is the test accuracy of testing data $\mathrm{f}$ in the classifier which is built with the feature selection of training data. The classification accuracy of minimum distance classifier is given in Equation (6).

$$
\operatorname{Accuracy}(\mathrm{f})=\frac{\mathrm{s}}{\mathrm{t}} * 100
$$

s: Number of samples that are correctly classified in test data by minimum distance classifier or $\mathrm{k}-\mathrm{NN}$ Classifier

$\mathrm{t}$ : Total number of samples in test data

Thus the important and essential features are selected by using ECSO more efficiently from the given dataset.

\section{Parameter $\mathrm{Pa}$}

In enhanced cuckoo search the $\mathrm{P}_{\mathrm{a}}$ value is changed dynamically by using the formula given in Equation

(7)

$$
\mathrm{P}_{\mathrm{a}}=\mathrm{P}_{\mathrm{a}} \max -\frac{\mathrm{P}_{\mathrm{a}} \max -\mathrm{P}_{\mathrm{a}} \min }{\text { iter_max }} * \text { iter }
$$

\section{Algorithm 2: ECSO}

Generate an initial population of $\mathrm{n}$ host nests with $\mathrm{m}$ eggs (tweets);

while ( $\mathrm{t}<$ MaxGeneration) or (stop criterion)

for every nest

Get a cuckoo type randomly (say i)

Check the type of the cuckoo

If cuckoo_type=common_cuckoo

Create two eggs through crossover with two best eggs in the nest (solution) and choose the best one among them

Else if cuckoo_type = European cuckoo

Create two eggs through crossover with uniform mutation operator with any two eggs in the nest

Select the best one among them

Else

Create egg with random solution (cryptic egg)

End if

Evaluate its fitness fi using (5)

Select an egg with the worst solution in the nest (say j)
If (fi>fj)

Replace $\mathrm{j}$ by new solution $\mathrm{i}$

End if

Rank the egg based on the solution

Find the best solution among $\mathrm{m}$ eggs in the nest

Abandon a fraction of the eggs in the nest which have the worst solutions and built new ones using levy flight (3) and (4)

Compute accuracy value using (6)

Update parameter $\mathrm{P}_{\mathrm{a}}$ using (7)

Keep the best solutions

End for

Rank the eggs in all nests using fitness value and find the current best

Select the more relevant and important tweets

End while

The algorithm describes that the Tweets (features) are selected by using best fitness values of objective function. The levy flight is built the new best solutions and unnecessary features are reduced significantly. Thus the ECSO optimization algorithm is used to increase the more informative features for the given Twitter dataset.

\section{Cyberbullying detection by using Hybrid Firefly Artificial Neural Network (HFANN) algorithm}

In this work, Cyberbullying detection is performed by using Hybrid Firefly Artificial Neural Network (HFANN) algorithm. As cyberbullying is considered a classification issue (i.e., categorizing an instance as offensive or non-offensive), HFANN is employed in this work for the further evolution of their classification accuracy and performance in detecting cyberbullying on Twitter. It is used to provide more accurate result for the Cyberbullying detection analysis. Artificial Neural Network (ANN) is also one of biological inspired technology and ANN models the human brain. Imitating the behaviour of the neurons of a brain, an ANN is designed as a connection of nodes, neurons, dendrites and synapses connected with the help of arcs, just like a biological 
nervous connection. A weight is associated with every arc. An activation function is applied on these arcs to adjust the weights after applying inputs to get a desired set of outputs. A neural network is basically an implementation of machine learning in the form a computing model [22].

ANN is capable of performing perceptual and recognition tasks in lesser amount of time. In this work, ANN is used to classify whether the tweet is cyberbullying or non-cyberbullying. A neural network exploits the non-linearity of a problem to define a set of desired inputs. Fig 4 shows the structure of ANN. It consists three components, in the form of layers.

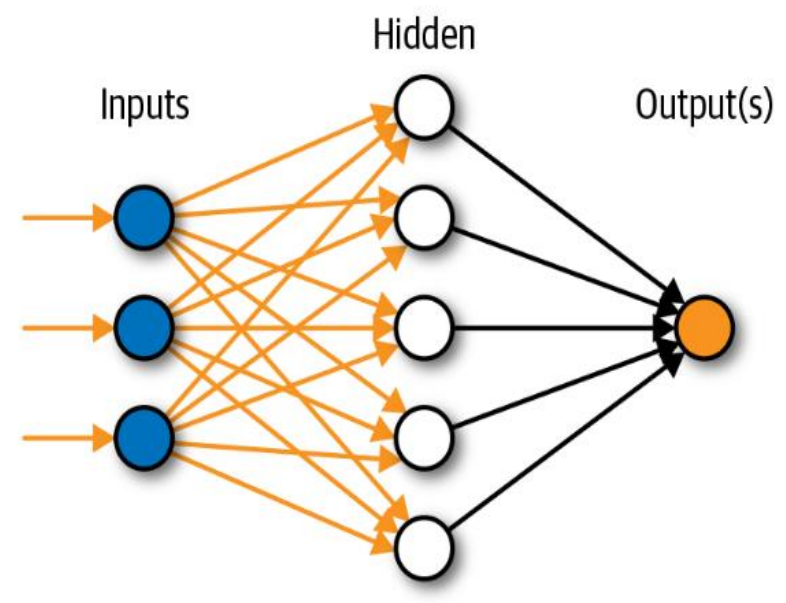

Fig 4. Structure of ANN

Input Layer - Input layer bears the information that is fed into the network. This information is rather raw, initially.

Hidden Layer - Hidden layer's basic job is to process the raw information received from the input layer into something that can be used by the output layer.ANN architecture can have one and more hidden layers.

Output Layer - Information received from the hidden layer is fed to the output layer and it is processed to produce the desired results
ANN is characterized by containing adaptive weights along paths between neurons that can be tuned by a learning algorithm that learns from observed data in order to improve the model. In addition to the learning algorithm itself, one must choose an appropriate cost function. For cyberbullying detection, they used word embedding that makes a list of predefined insulting words and assign weights to obtain bullying feature.

The cost function is what's used to learn the optimal solution to the problem being solved. This involves determining the best values for all of the tunable model parameters, with neuron path adaptive weights being the primary target, along with algorithm tuning parameters such as the learning rate. It's usually done through optimization techniques such as gradient descent or stochastic gradient descent.

In the hidden layer, the features from the input layer are calculated by a linear function (8) and a transfer function (9) to generate the output feature of a hidden node

$$
\mathrm{N}_{\mathrm{i}}=\sum_{\mathrm{p}=1}^{\mathrm{P}} \mathrm{W}_{\mathrm{i}, \mathrm{p}} \mathrm{I}_{\mathrm{P}}+\mathrm{B}_{\mathrm{i}}
$$

where $N_{i}$ is the value of the $i^{\text {th }}$ feature; $w_{i, p}$ and $B_{i}$ are the weight of the $\mathrm{p}^{\text {th }}$ input to the $\mathrm{i}^{\text {th }}$ hidden node and the bias parameter of the $\mathrm{i}^{\text {th }}$ hidden node, respectively; $I_{P}$ is the value of the $\mathrm{p}^{\text {th }}$ input node.

The transfer function is defined as:

$$
\mathrm{y}_{\mathrm{i}}=\mathrm{f}\left(\mathrm{N}_{\mathrm{i}}\right)=\frac{1}{1+\exp \left(-\mathrm{N}_{\mathrm{i}}\right)}
$$

Where the transfer function in this study is a sigmoid function and $y_{i}$ is the output signal of the $i^{\text {th }}$ hidden node

During the learning process, ANN uses the Mean Square Error (MSE), to evaluate the performance of the model as follows:

$$
\text { MSE }=\frac{1}{\mathrm{NN}_{\text {out }}} \sum_{\mathrm{n}=1}^{\mathrm{N}} \sum_{\mathrm{o}=1}^{\mathrm{N}_{\text {out }}}\left(\mathrm{e}_{\mathrm{n}, \mathrm{o}}\right)^{2}
$$

where $\mathrm{N}$ and $\mathrm{N}_{\text {out }}$ are the number of instances and the number of outputs, respectively; $\mathrm{e}_{\mathrm{n}, \mathrm{o}}$ is the training error at the $\mathrm{o}^{\text {th }}$ output with $\mathrm{n}^{\text {th }}$ instance; $\mathrm{y}$ is the actual output and $\bar{y}$ is the predicted output by ANN. 
To reduce the error rate and increase the accuracy, firefly algorithm is hybrid with ANN. The optimization technique basically try to make the ANN solution be as close as possible to the optimal solution, which when successful means that the ANN is able to solve the intended problem with high performance. In this research, firefly algorithm is combined with ANN algorithm to improve the convergence speed and accuracy for the given Twitter dataset.

Firefly algorithm [23] is inspired by biochemical and social aspects of real fireflies. Real fireflies produce a short and rhythmic flash that helps them in attracting (communicating) their mating partners and also serves as protective warning mechanism. FA formulates this flashing behavior with the objective function of the problem to be optimized. The following three rules are idealized for basic formulation of firefly algorithm (3) all fireflies are unisex so that fireflies will attract each other regardless of their sex. (2) Attractiveness is proportional to their brightness, which decreases as distance increases between two flies. Thus the less bright one will move towards the brighter one. In case it is unable to detect brighter one it will move randomly. (3) The brightness of a firefly is determined by the landscape of the objective function. $x_{i}=x_{i}+\beta_{o} \times e^{-\gamma r^{2} i j} \times\left(x_{j}-x_{i}\right)+\alpha \times($ rand -0.5$)$

Where the first part of (1) represents the movement of attraction between two fireflies, the second part represents the attraction. $\beta_{0}$ is the initial attractiveness which is always set to 1 , and $\gamma$ is the absorption coefficient which controls the speed of convergence between fireflies. The third part of (1) is randomization, where $\alpha$ is a constant randomization parameter defined between $[0,1]$, it represents the noise of the environment that be used to provide more diversity of solutions, rand is a random number generated from a uniform distribution $[0,1]$ and adjusted to range between $[-0.5,0.5]$ by expression (rand - 0.5). The proposed method in Twitter is analyzed as positive opinion tweets and negative opinion tweets

\section{Algorithm 3: HFANN}

Input: Twitter dataset, training data, testing data

Objective function: Assign the Cyberbullying detection accuracy as fitness function

Output: Cyberbullying or Non Cyberbullying Tweets

1. Start

2. For each training data $\{(\mathrm{x}(\mathrm{i}), \mathrm{d}(\mathrm{i})), \mathrm{i}=1, \ldots \mathrm{p}$

3. Compute weight value and bias for every feature in input data

4. Adjust weight value and bias value for every feature

5. Objective function $f(x), x=(x 1, \ldots \ldots x 4) T$

6. Generate initial population of fireflies $\mathrm{xi}$ $(\mathrm{i}=1,2, \ldots \mathrm{n})$

7. Light intensity Ii at $x i$ is determined by $f(x i)$

8. Define light absorption coefficient $\gamma$

9. for $\mathrm{i}=1: \mathrm{n}$ all $\mathrm{n}$ fireflies

10. for $\mathrm{j}=1: \mathrm{i}$ all $\mathrm{n}$ fireflies

11. if $(\mathrm{Ij}>\mathrm{Ii})$,

12. end if

13. Evaluate new solution and update light intensity

14. end for $\mathrm{j}$

15. end for $\mathrm{i}$

16. Rank the fireflies and find the current best

17. Compute best fitness values using (11)

18. Update weight and bias value(8)

19. End for

20. $A N N=A N N($ Input, Hidden, Output $)$

21. Train model $=$ Accuracy (train feature)

22. Test model = Accuracy (test feature)

23. Calculate error rate (MSE) using (10)

24. MSE( train and test tweets)

25. Return more accurate results (cyberbullying or non-cyberbullying tweet)

By achieving the higher accuracy, this method improves cyberbullying detection to help people to use social media safely. It is used for detecting 
cyberbullying pattern for the large size of training data.

\section{EXPERIMENTAL RESULT}

In Twitter dataset 4556 tweets from various topics such as demonetisation, kids, mobilephones, sachin and whatsapp words are searched and analyzed as positive opinion tweets and negative opinion tweets. The analyzed tweets are presented in Table 1 . The performance metrics are considered such as precision, accuracy, recall, f-measure and time complexity. The existing algorithms of SSVM, $\mathrm{v}$ and the proposed ECSO+HFANN algorithm is evaluated to perform the above mentioned metrics on the Twitter dataset

Table 1 Collected Tweets with various search terms and opinion analysis

\begin{tabular}{|l|l|l|l|}
\hline \multirow{2}{*}{ File Name } & Total & \multicolumn{2}{|l|}{ Actual } \\
\cline { 3 - 4 } & $\begin{array}{l}\text { No. of } \\
\text { Tweets }\end{array}$ & $\begin{array}{l}\text { Positive } \\
\text { Opinion } \\
\text { Tweets }\end{array}$ & $\begin{array}{l}\text { Negative } \\
\text { Opinion } \\
\text { Tweets }\end{array}$ \\
\hline demonetisation.txt & 1003 & 498 & 505 \\
\hline kids.txt & 984 & 402 & 582 \\
\hline mobilephones.txt & 783 & 599 & 184 \\
\hline sachin.txt & 994 & 483 & 511 \\
\hline whatsapp.txt & 792 & 599 & 193 \\
\hline
\end{tabular}

- $\quad$ True Positive (TP) $\rightarrow$ Correctly identified as positive opinion tweets

- $\quad$ False Positive (FP) $\rightarrow$ Incorrectly identified as positive opinion tweets

- $\quad$ True Negative (TN) $\rightarrow$ Correctly identified as negative opinion tweets

- $\quad$ False Negative (FP) $\rightarrow$ Incorrectly identified s negative opinion tweets

\section{Precision}

The precision is calculated as follows:

Precision $=\quad \frac{\text { True positive }}{\text { True positive+False positive }}$
Precision can be view as a computation of accuracy or quality, whereas recall is a measure of completeness or quantity. In general, high precision indicates that an algorithm returned substantially more relevant results than irrelevant. In a classification task, the precision for a class is the number of true positives divided by the total number of elements labeled as belonging to the positive class.

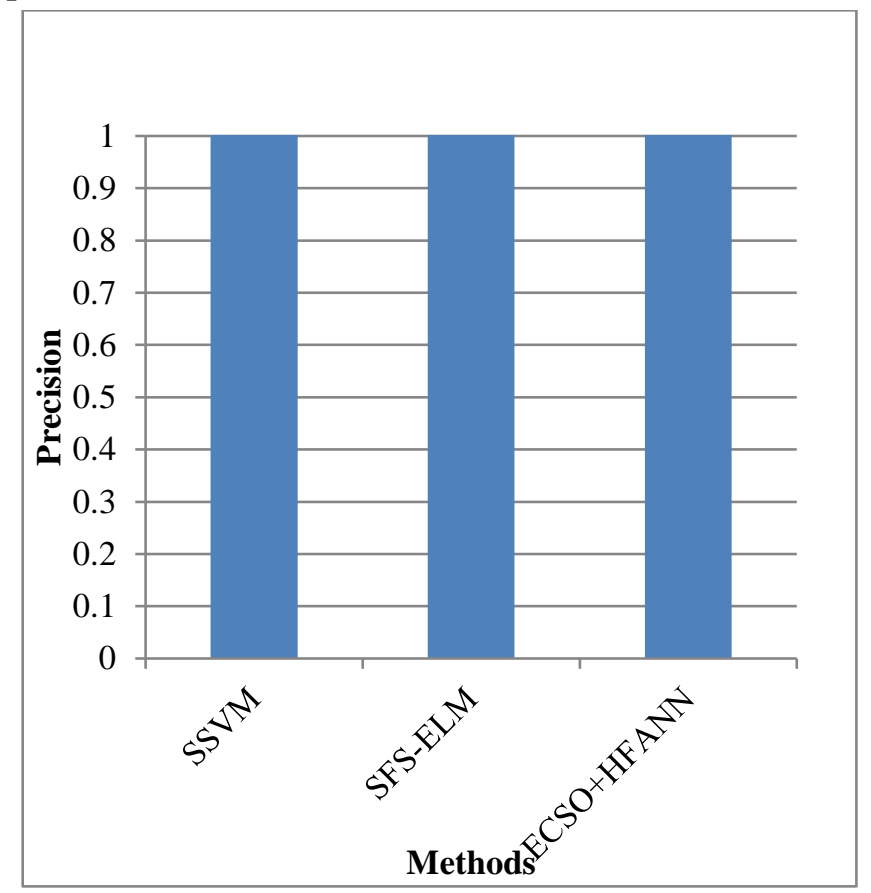

Fig 5 Precision

From the above Fig 5, it can be observed that the comparison metric is evaluated using existing and proposed method in terms of precision. For $\mathrm{x}$-axis the methods are taken and in $\mathrm{y}$-axis the precision value is plotted. The existing methods are such as SSVM and SFS-ELM algorithms provide lower precision whereas proposed ECSO+HFANN algorithm provides higher precision for the given Twitter dataset. Thus the result concludes that the proposed ECSO+HFANN increase the Cyberbullying detection accuracy through the optimal selection of features.

\section{Recall}

The calculation of the recall value is done as follows:

$$
\text { Recall }=\frac{\text { True positive }}{\text { True positive }+ \text { False negative }}
$$

The comparison graph is depicted as follows: 
Recall is defined as the number of relevant documents retrieved by a search divided by the total number of existing relevant documents, while precision is defined as the number of relevant documents retrieved by a search divided by the total number of documents retrieved by that search.

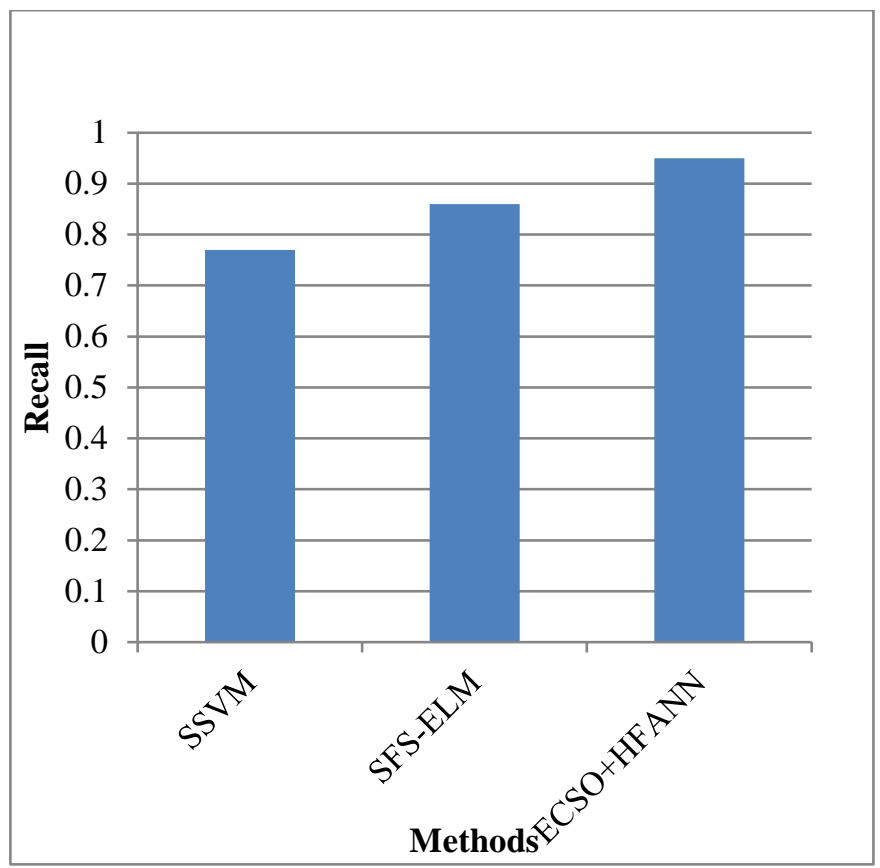

Fig 6 Recall

From the above Fig 6, it can be observed that the comparison metric is evaluated using existing and proposed method in terms of recall. For $\mathrm{x}$-axis the methods are taken and in y-axis the recall value is plotted. The existing methods are such as SSVM and SFS-ELM algorithms provide lower recall whereas proposed ECSO+HFANN algorithm provides higher recall for the given Twitter dataset. Thus the result concludes that the proposed ECSO+HFANN increase the Cyberbullying detection accuracy through the optimal selection of features.

\section{F-measure}

F1-score is defined as:

$$
\mathrm{F} 1-\text { score }=\frac{2 \times \text { precision } \times \text { recall }}{\text { precision }+ \text { recall }}
$$

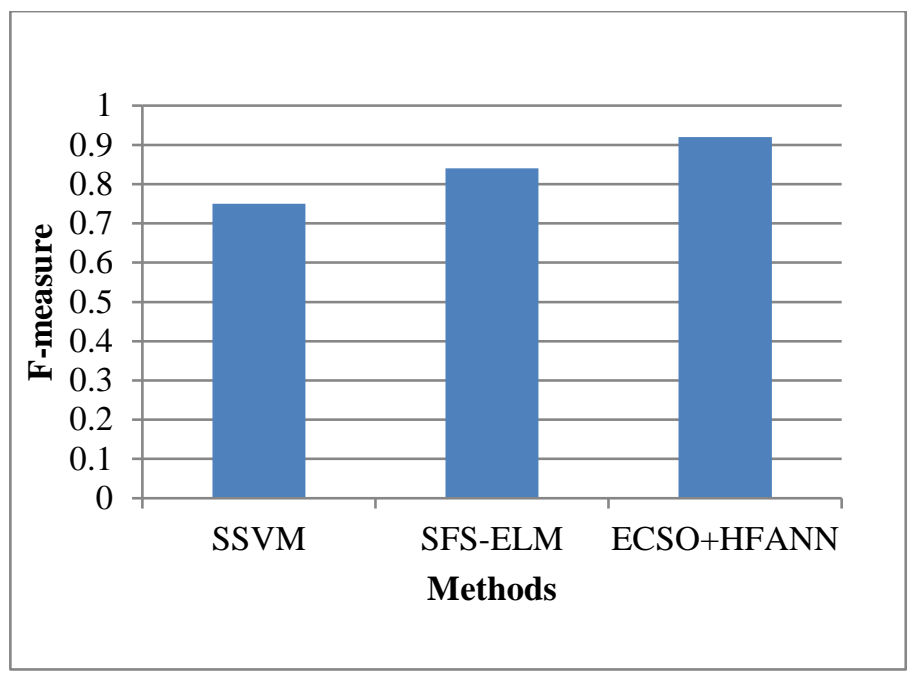

Fig 7 F-measure

From the above Fig 7, it can be observed that the comparison metric is evaluated using existing and proposed method in terms of $\mathrm{F}$-measure. For $\mathrm{x}$-axis the methods are taken and in y-axis the F-measure value is plotted. The existing methods are such as SSVM and SFS-ELM algorithms provide lower Fmeasure whereas proposed ECSO+HFANN algorithm provides higher F-measure for the given Twitter dataset. Thus the result concludes that the proposed ECSO+HFANN increase the Cyberbullying detection accuracy through the optimal selection of features.

\section{Accuracy}

Accuracy is determined as the overall correctness of the model and is computed as the total actual classification parameters $\left(T_{p}+T_{n}\right)$ which is segregated by the sum of the classification parameters $\left(T_{p}+T_{n}+F_{p}+F_{n}\right)$. The accuracy is computed as like :

$$
\text { Accuracy }=\frac{T_{p}+T_{n}}{\left(T_{p}+T_{n}+F_{p}+F_{n}\right)}
$$




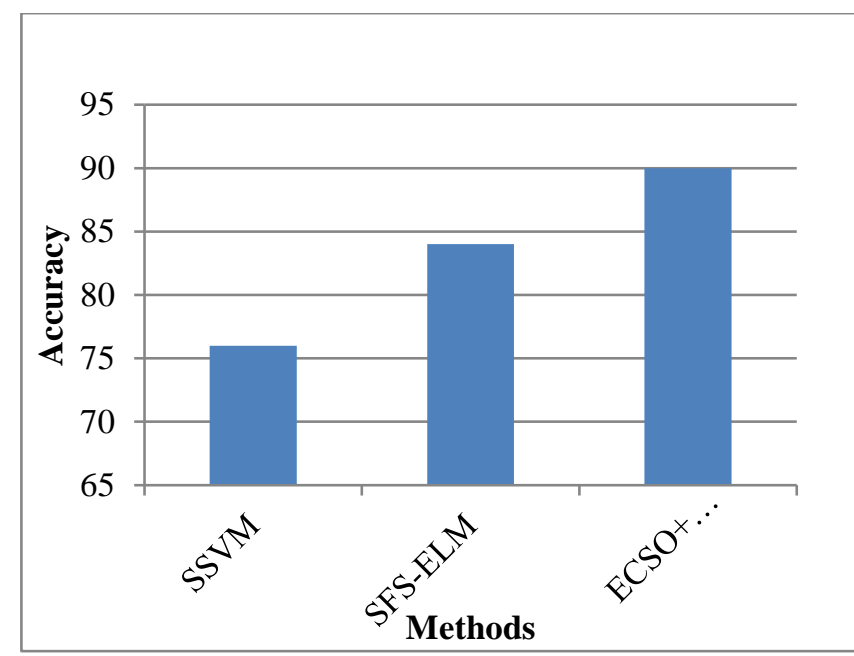

Fig 8 Accuracy

From the above Fig 8 , it can be observed that the comparison metric is evaluated using existing and proposed method in terms of accuracy. For $\mathrm{x}$-axis the methods are taken and in y-axis the accuracy value is plotted. The existing methods are such as SSVM and SFS-ELM algorithms provide lower accuracy whereas proposed ECSO+HFANN algorithm provides higher accuracy for the given Twitter dataset. Thus the result concludes that the proposed ECSO+HFANN increases the Cyberbullying detection accuracy through the optimal selection of features

\section{Time complexity}

The system is better when the proposed algorithm executes in less time consumption.

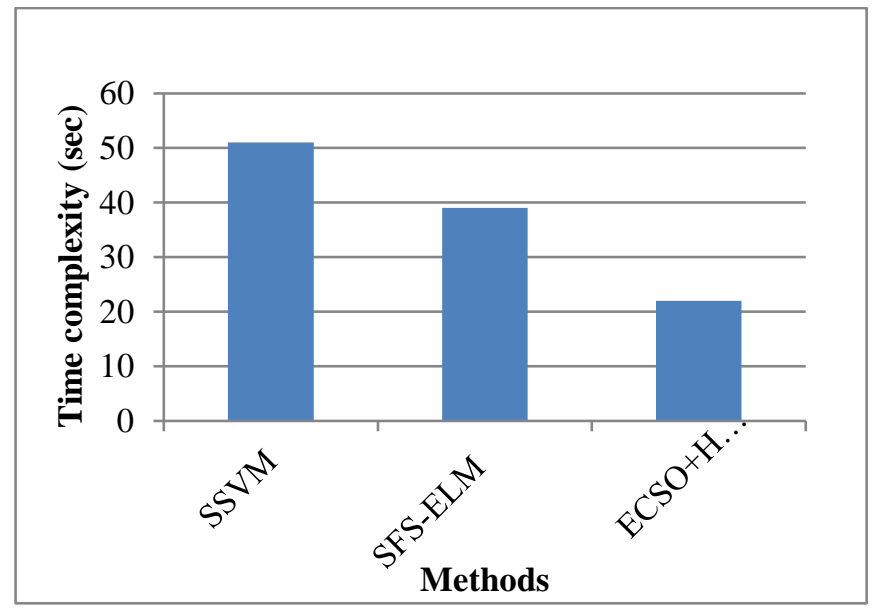

Fig 9. Time complexity comparison

From the above Fig 9, it can be observed that the comparison metric is evaluated using existing and proposed method in terms of time complexity. For $\mathrm{x}^{-}$ axis the methods are taken and in y-axis the time complexity value is plotted. The existing methods are such as SSVM and SFS-ELM algorithms provide higher time complexity whereas proposed ECSO+HFANN algorithm provides lower time complexity for the given Twitter dataset. Thus the result concludes that the proposed ECSO+HFANN increases the Cyberbullying detection performance by classifying accurate results in Twitter dataset.

\section{CONCLUSION}

In this research work, ECSO+HFANN algorithm is proposed to improve the Cyberbullying detection accuracy results for the given Twitter dataset prominently. In this work, the preprocessing is done by using $\mathrm{k}$-means clustering algorithm. It is used to increase the Cyberbullying detection accuracy by reducing the missing values and redundancy features from the given Twitter dataset. It improves the quality of the research data and subsequent analytical steps, this includes removing stop words, extra characters and hyperlinks. Then these features are taken into feature selection process. The subset selection of features is performed by using ECSO optimization algorithm. It is used to select the important and relevant features by using best fitness function of cuckoos. Then the classification is done by HFANN algorithm which is used to increase the Cyberbullying detection accuracy in higher. The result concludes that the proposed ECSO+HFANN algorithm provides higher Cyberbullying detection performance in terms of greater precision, recall, $\mathrm{f}$ measure and accuracy values and lower time complexity than the existing SSVM and SFS-ELM algorithms. In future, optimization based fuzzy clustering algorithm can be developed for handling the huge Twitter dataset. 


\section{REFERENCES}

[1]. Velez, D., Sueiras, J., Ortega, A., \& Velez, J. F. (2015). A method for K-Means seeds generation applied to text mining. Statistical Methods \& Applications, 1-23

[2]. Reynolds, Kelly Edwards, April Edwards, Lynne. (2011). Using Machine Learning to Detect Cyberbullying. Proceedings - 10th International Conference on Machine Learning and Applications, ICMLA 2011. 2. 10.1109/ICMLA.2011.152.

[3]. Van Hee C, Jacobs G, Emmery C, et al. Automatic detection of cyberbullying in social media text. PLoS One. 2018;13(10):e0203794

[4]. D. Terrana, A. Augello, G. Pilato, "Automatic Unsupervised Polarity Detection on a Twitter Data Stream" in Semantic Computing (ICSC),2014 IEEE International Conference, pp.128-134, 16-18 June 2014.

[5]. Özel, Selma Ayşe, et al. "Detection of cyberbullying on social media messages in Turkish." 2017 International Conference on Computer Science and Engineering (UBMK). IEEE, 2017

[6]. Fouad, Mohammed M., Tarek F. Gharib, and Abdulfattah S. Mashat. "Efficient twitter sentiment analysis system with feature selection and classifier ensemble." International Conference on Advanced Machine Learning Technologies and Applications. Springer, Cham, 2018.

[7]. Wang, Xiaolong, et al. "Topic sentiment analysis in twitter: a graph-based hashtag sentiment classification approach." Proceedings of the 20th ACM international conference on Information and knowledge management. 2011.

[8]. Al-Garadi, Mohammed Ali, Kasturi Dewi Varathan, and Sri Devi Ravana. "Cybercrime detection in online communications: The experimental case of cyberbullying detection in the Twitter network." Computers in Human Behavior 63 (2016): 433-443.

[9]. León-Paredes, Gabriel A., et al. "Presumptive Detection of Cyberbullying on Twitter through Natural Language Processing and Machine Learning in the Spanish Language." 2019 IEEE CHILEAN Conference on Electrical, Electronics Engineering, Information and Communication Technologies (CHILECON). IEEE, 2019.

[10]. Zhang, Jianwei, et al. "Cyberbullying Detection on Twitter using Multiple Textual Features." 2019 IEEE 10th International Conference on Awareness Science and Technology (iCAST). IEEE, 2019.

[11]. Balakrishnan, Vimala, Shahzaib Khan, and Hamid R. Arabnia. "Improving cyberbullying detection using Twitter users' psychological features and machine learning." Computers \& Security 90 (2020): 101710.

[12]. Dash, B., et al. "A hybridized K-means clustering approach for high dimensional dataset." International Journal of Engineering, Science and Technology 2.2 (2010): 59-66.

[13]. Salesi, Sadegh, and Georgina Cosma. "A novel extended binary cuckoo search algorithm for feature selection." 2017 2nd international conference on knowledge engineering and applications (ICKEA). IEEE, 2017.

[14]. Abd El Aziz, Mohamed, and Aboul Ella Hassanien. "Modified cuckoo search algorithm with rough sets for feature selection." Neural Computing and Applications 29.4 (2018): 925934.

[15]. Emary, Eid, et al. "Firefly optimization algorithm for feature selection." Proceedings of the 7th Balkan Conference on Informatics Conference. 2015.

[16]. Ghiassi, Manoochehr, James Skinner, and David Zimbra. "Twitter brand sentiment analysis: A hybrid system using n-gram analysis and dynamic artificial neural network." Expert 
Systems with applications 40.16 (2013): 62666282.

[17]. Mohamad, Ismail Bin, and Dauda Usman. "Standardization and its effects on K-means clustering algorithm." Research Journal of Applied Sciences, Engineering and Technology 6.17 (2013): 3299-3303.

[18]. Al Shalabi, Luai, and Zyad Shaaban. "Normalization as a preprocessing engine for data mining and the approach of preference matrix." 2006 International conference on dependability of computer systems. IEEE, 2006.

[19]. H. Hosseinmardi, S. A. Mattson, R. I. Raq, R. Han, Q. Lv, and S. Mishra. AnalyzingLabeled Cyberbullying Incidents on the Instagram Social Network. InIn SocInfo,2015

[20]. Huang, Li, et al. "Chaos-enhanced Cuckoo search optimization algorithms for global optimization." Applied Mathematical Modelling 40.5-6 (2016): 3860-3875.

[21]. Kamoona, Ammar Mansoor, Jagdish Chandra Patra, and Alex Stojcevski. "An enhanced cuckoo search algorithm for solving optimization problems." 2018 IEEE Congress on Evolutionary Computation (CEC). IEEE, 2018.

[22]. Jalali, Seyed Mohammad Jafar, et al. "Evolving artificial neural networks using butterfly optimization algorithm for data classification." International Conference on Neural Information Processing. Springer, Cham, 2019.

[23]. Selvakumar, B., and Karuppiah Muneeswaran. "Firefly algorithm based feature selection for network intrusion detection." Computers \& Security 81 (2019): 148-155.

\section{Cite this article as :}

Sherly T. T., Dr. B. Rosiline Jeetha, "Enhanced Cuckoo Search Optimization and Hybrid Firefly Artificial Neural Network Algorithm for Cyberbullying Detection on Twitter Dataset", International Journal of Scientific Research in Computer Science, Engineering and Information Technology (IJSRCSEIT), ISSN : 2456-3307, Volume 7 Issue 4, pp. 455-470, July-August 2021. Available at doi : https://doi.org/10.32628/CSEIT217486 Journal URL : https://ijsrcseit.com/CSEIT217486 